\title{
General Integral Operator of Analytic Functions Involving Functions with Positive Real Part
}

\author{
B. A. Frasin \\ Department of Mathematics, Faculty of Science, Al al-Bayt University, P.O. Box 130095, Mafraq, Jordan
}

Correspondence should be addressed to B. A. Frasin; bafrasin@yahoo.com

Received 22 August 2012; Accepted 21 October 2012

Academic Editor: S. T. Ali

Copyright $\odot 2013$ B. A. Frasin. This is an open access article distributed under the Creative Commons Attribution License, which permits unrestricted use, distribution, and reproduction in any medium, provided the original work is properly cited.

Let $B_{\beta}$ be the integral operator defined by $B_{\beta}(z)=\left[\beta \int_{0}^{z} t^{\beta-1} \prod_{i=1}^{n}\left[\left(\left(f_{i}(t)\right) / t\right)^{\gamma_{i}} p_{i}^{\zeta_{i}}(t)\right] d t\right]^{1 / \beta}$ where each of the functions $f_{i}$ and $p_{i}$ are, respectively, analytic functions and functions with positive real part defined in the open unit disk for all $i=1, \ldots, n$. The object of this paper is to obtain several univalence conditions for this integral operator. Our main results contain some interesting corollaries as special cases.

\section{Introduction and Definitions}

Let $\mathscr{A}$ denote the class of the normalized functions of the form

$$
f(z)=z+\sum_{k=2}^{\infty} a_{k} z^{k}
$$

which are analytic in the open unit disk $\mathscr{U}=\{z \in \mathbb{C}:|z|<$ $1\}$. Further, by $\delta$ we shall denote the class of all functions in $\mathscr{A}$ which are univalent in $\mathscr{U}$. Also, let $\mathscr{P}$ be the class of all functions which are analytic in $\mathscr{U}$ and satisfy $p(0)=$ $1, \operatorname{Re}\{p(z)\}>0$.

Frasin and Darus [1] (see also [2]) defined the family $\mathscr{B}(\delta), 0 \leq \delta<1$ so that it consists of functions $f \in \mathscr{A}$ satisfying the condition

$$
\left|\frac{z^{2} f^{\prime}(z)}{f^{2}(z)}-1\right|<1-\delta(z \in \mathcal{U})
$$

Very recently many authors studied the problem of integral operators which preserve the class $\mathcal{S}$ (see, e.g., [3$15])$. In this paper, we obtain new sufficient conditions for the univalence of the general integral operator $B_{\beta}(z)$ defined by

$$
B_{\beta}(z)=\left[\beta \int_{0}^{z} t^{\beta-1} \prod_{i=1}^{n}\left[\left(\frac{f_{i}(t)}{t}\right)^{\gamma_{i}} p_{i}^{\zeta_{i}}(t)\right] d t\right]^{1 / \beta},
$$

where $f_{i} \in \mathscr{A}, p_{i} \in \mathscr{P}, \beta \in \mathbb{C}^{*}=\mathbb{C} \backslash\{0\}$, and $\gamma_{i}, \zeta_{i} \in \mathbb{C}$ for all $i=1, \ldots, n$.

Here and throughout in the sequel, every many-valued function is taken with the principal branch.

Remark 1. Note that the integral operator $B_{\beta}$ generalizes the following operators introduced and studied by several authors:

(1) If we let $\zeta_{i}=0$, for all $i=1, \ldots, n$, in (3), we obtain the integral operator:

$$
\begin{aligned}
I_{\gamma} & \left(f_{1}, \ldots, f_{n}\right)(z) \\
& =\left\{\beta \int_{0}^{z} t^{\beta-1}\left(\frac{f_{1}(t)}{t}\right)^{\gamma_{1}} \cdots\left(\frac{f_{n}(t)}{t}\right)^{\gamma_{n}} d t\right\}^{1 / \beta},
\end{aligned}
$$

introduced and studied by D. Breaz and N. Breaz [16].

(2) If we let $\gamma_{i}=0$, for all $i=1, \ldots, n$, in (3), we obtain the integral operator:

$$
\begin{aligned}
I_{\beta}( & \left.p_{1}, \ldots, p_{n} ; \alpha_{1}, \ldots, \alpha_{n}\right)(z) \\
\quad & \left\{\int_{0}^{z} \beta t^{\beta-1}\left(p_{1}(t)\right)^{\zeta_{1}} \cdots\left(p_{n}(t)\right)^{\zeta_{n}} d t\right\}^{1 / \beta}
\end{aligned}
$$

introduced and studied by Frasin [17]. 
(3) If we let $\beta=1$ and $\zeta_{i}=0$, for all $i=1, \ldots, n$, in (3), we obtain the integral operator:

$$
F_{n}(z)=\int_{0}^{z}\left(\frac{f_{1}(t)}{t}\right)^{\gamma_{1}} \cdots\left(\frac{f_{n}(t)}{t}\right)^{\gamma_{n}} d t,
$$

introduced and studied by D. Breaz and N. Breaz [16].

In order to derive our main results, we have to recall here the following lemmas.

Lemma 2 (see [18]). Let $\alpha \in \mathbb{C}$ with $\operatorname{Re}(\alpha)>0$. If $h \in \mathscr{A}$ satisfies

$$
\frac{1-|z|^{2 \operatorname{Re}(\alpha)}}{\operatorname{Re}(\alpha)}\left|\frac{z h^{\prime \prime}(z)}{h^{\prime}(z)}\right| \leq 1
$$

for all $z \in \mathcal{U}$, then, for any complex number $\beta$ with $\operatorname{Re}(\beta) \geq$ $\operatorname{Re}(\alpha)$, the integral operator

$$
F_{\beta}(z)=\left\{\beta \int_{0}^{z} t^{\beta-1} h^{\prime}(t) d t\right\}^{1 / \beta}
$$

is in the class $\mathcal{S}$.

Lemma 3 (see [13]). Let $\beta \in \mathbb{C}$ with $\operatorname{Re}(\beta)>0, c \in \mathbb{C}$ with $|c| \leq 1, c \neq-1$. If $h \in \mathscr{A}$ satisfies

$$
\left.|c| z\right|^{2 \beta}+\left(1-|z|^{2 \beta}\right) \frac{z h^{\prime \prime}(z)}{\beta h^{\prime}(z)} \mid \leq 1,
$$

for all $z \in \mathcal{U}$, then the integral operator $F_{\beta}(z)$ defined by (8) is in the class $\mathcal{S}$.

Lemma 4 (see [19]). If $p(z) \in \mathscr{P}$, then we have

$$
\left|\frac{z p^{\prime}(z)}{p(z)}\right|<\frac{2|z|}{1-|z|^{2}}, \quad(z \in \mathcal{U}) \text {. }
$$

Lemma 5 (see [20]). If $f(z) \in \mathscr{B}(\delta)$, then

$$
\left|\frac{z f^{\prime}(z)}{f(z)}-1\right|<\frac{(1-\delta)(1+|z|)}{1-|z|} \quad(z \in \mathcal{U}) \text {. }
$$

Also, we need the following general Schwarz Lemma.

Lemma 6 (see [21]). Let the function $f$ be regular in the disk $\mathcal{U}_{R}=\{z:|z|<R\}$, with $|f(z)|<M$ for fixed $M$. If $f(z)$ has one zero with multiplicity order bigger than $m$ for $z=0$, then

$$
|f(z)| \leq \frac{M}{R^{m}}|z|^{m} \quad\left(z \in \mathscr{U}_{R}\right) .
$$

The equality can hold only if

$$
f(z)=e^{i \theta}\left(\frac{M}{R^{m}}\right) z^{m}
$$

where $\theta$ is constant.

\section{Univalence Conditions for the Operator $B_{\beta}$}

We first prove the following theorem.

Theorem 7. Let $f(z) \in \mathscr{B}\left(\delta_{i}\right) ; 0 \leq \delta_{i}<1$ and $p_{i}(z) \in \mathscr{P}$ for all $i=1, \ldots, n$. Let $\alpha \in \mathbb{C}$ with $\operatorname{Re}(\alpha)=a>0$. If

$$
\sum_{i=1}^{n}\left[\left|\gamma_{i}\right|\left(1-\delta_{i}\right)+\left|\zeta_{i}\right|\right] \leq \min \left\{\frac{a}{2} ; \frac{1}{4}\right\},
$$

then the integral operator $B_{\beta}(z)$ defined by (3) is in the class $\mathcal{S}$.

Proof. Define the regular function $h(z)$ by

$$
h(z)=\int_{0}^{z} \prod_{i=1}^{n}\left[\left(\frac{f_{i}(t)}{t}\right)^{\gamma_{i}} p_{i}^{\zeta_{i}}(t)\right] d t .
$$

Then it is easy to see that

$$
h^{\prime}(z)=\prod_{i=1}^{n}\left[\left(\frac{f_{i}(z)}{z}\right)^{\gamma_{i}} p_{i}^{\zeta_{i}}(z)\right],
$$

and $h(0)=h^{\prime}(0)-1=0$. Differentiating both sides of (16) logarithmically, we obtain

$$
\frac{z h^{\prime \prime}(z)}{h^{\prime}(z)}=\sum_{i=1}^{n} \gamma_{i}\left(\frac{z f_{i}^{\prime}(z)}{f_{i}(z)}-1\right)+\sum_{i=1}^{n} \zeta_{i} \frac{z p_{i}^{\prime}(z)}{p_{i}(z)} .
$$

Thus, we have

$$
\left|\frac{z h^{\prime \prime}(z)}{h^{\prime}(z)}\right| \leq \sum_{i=1}^{n}\left|\gamma_{i}\right|\left|\frac{z f_{i}^{\prime}(z)}{f_{i}(z)}-1\right|+\sum_{i=1}^{n}\left|\zeta_{i}\right|\left|\frac{z p_{i}^{\prime}(z)}{p_{i}(z)}\right| .
$$

Since $f_{i}(z) \in \mathscr{B}\left(\delta_{i}\right)$ and $p_{i}(z) \in \mathscr{P}$ for all $i=1, \ldots, n$, from (18), (11), and (10), we obtain

$$
\begin{aligned}
\left|\frac{z h^{\prime \prime}(z)}{h^{\prime}(z)}\right| & \leq \sum_{i=1}^{n}\left|\gamma_{i}\right|\left(1-\delta_{i}\right)\left(\frac{1+|z|}{1-|z|}\right)+\sum_{i=1}^{n}\left|\zeta_{i}\right|\left(\frac{2|z|}{1-|z|^{2}}\right) \\
& \leq \sum_{i=1}^{n}\left|\gamma_{i}\right|\left(1-\delta_{i}\right)\left(\frac{2}{1-|z|}\right)+\sum_{i=1}^{n}\left|\zeta_{i}\right|\left(\frac{2}{1-|z|}\right) .
\end{aligned}
$$

Multiply both sides of (19) by $\left(1-|z|^{2 \operatorname{Re}(\alpha)}\right) / \operatorname{Re}(\alpha)$, we get

$$
\begin{aligned}
\frac{1-|z|^{2 \operatorname{Re}(\alpha)}}{\operatorname{Re}(\alpha)}\left|\frac{z h^{\prime \prime}(z)}{h^{\prime}(z)}\right| \leq & \frac{1-|z|^{2 \operatorname{Re}(\alpha)}}{1-|z|} \frac{2}{\operatorname{Re}(\alpha)} \\
& \times \sum_{i=1}^{n}\left[\left|\gamma_{i}\right|\left(1-\delta_{i}\right)+\left|\zeta_{i}\right|\right],
\end{aligned}
$$

for all $z \in \mathcal{U}$.

Let us denote $|z|=x, x \in[0,1), \operatorname{Re}(\alpha)=a>0$, and $\Phi(x)=\left(1-x^{2 a}\right) /(1-x)$. It is easy to prove that

$$
\Phi(x) \leq \begin{cases}1, & \text { if } 0<a<\frac{1}{2} \\ 2 a, & \text { if } \frac{1}{2}<a<\infty .\end{cases}
$$


From (20), (21), and the hypothesis (14), we have

$$
\begin{aligned}
& \frac{1-|z|^{2 a}}{a}\left|\frac{z h^{\prime \prime}(z)}{h^{\prime}(z)}\right| \\
& \quad \leq \begin{cases}\frac{2}{a} \sum_{i=1}^{n}\left[\left|\gamma_{i}\right|\left(1-\delta_{i}\right)+\left|\zeta_{i}\right|\right], & \text { if } 0<a<\frac{1}{2} \\
4 \sum_{i=1}^{n}\left[\left|\gamma_{i}\right|\left(1-\delta_{i}\right)+\left|\zeta_{i}\right|\right], & \text { if } \frac{1}{2}<a<\infty .\end{cases}
\end{aligned}
$$

$\leq 1$,

for all $z \in \mathcal{U}$. Applying Lemma 2 for the function $h(z)$, we prove that $B_{\beta}(z) \in \mathcal{S}$.

Letting $n=1, \delta_{1}=\delta, \gamma_{1}=\gamma, \zeta_{1}=\zeta$, and $f_{1}=f$ in Theorem 7, we obtain the following corollary.

Corollary 8. Let $f(z) \in \mathscr{B}(\delta) ; 0 \leq \delta<1$ and $p(z) \in \mathscr{P}$. Also, let $\alpha, \gamma, \zeta \in \mathbb{C}$ with $\operatorname{Re}(\alpha)=a>0$. If

$$
|\gamma|(1-\delta)+|\zeta| \leq \min \left\{\frac{a}{2} ; \frac{1}{4}\right\}
$$

then the integral operator $B_{\beta, \gamma, \zeta}(z)$ defined by

$$
B_{\beta, \gamma, \zeta}(z)=\left[\beta \int_{0}^{z} t^{\beta-1}\left(\frac{f(t)}{t}\right)^{\gamma} p^{\zeta}(t) d t\right]^{1 / \beta}
$$

is in the class $\mathcal{S}$.

If we set $\delta=0$ in Corollary 8 , we have the following.

Corollary 9. Let $f(z) \in \mathcal{S}$ and $p(z) \in p$. Also, let $\alpha, \gamma, \zeta \in \mathbb{C}$ with $\operatorname{Re}(\alpha)=a>0$. If

$$
|\gamma|+|\zeta| \leq \min \left\{\frac{a}{2} ; \frac{1}{4}\right\}
$$

then the integral operator $B_{\beta, \gamma, \zeta}(z)$ defined by (24) is in the class $\mathcal{S}$.

Next, we prove the following theorem.

Theorem 10. Let $f_{i}(z) \in \mathscr{A}$ satisfies $\operatorname{Re}\left(f_{i}(z) / z\right)>0$, and

$$
\left|\left(\gamma_{i}+\zeta_{i}\right)\left(\frac{z f_{i}^{\prime}(z)}{f_{i}(z)}-1\right)\right| \leq \frac{(2 a+1)^{(2 a+1) / 2 a}}{2 n},
$$

for all $i=1, \ldots, n$, where $\gamma_{i}, \zeta_{i}, \alpha \in \mathbb{C}$ with $\operatorname{Re}(\alpha)=a>0$, then the integral operator $B_{\beta}(z)$ defined by (3) is in the class $\mathcal{S}$.

Proof. Suppose that $\operatorname{Re}\left(f_{i}(z) / z\right)>0$ for all $i=1, \ldots, n$. Thus, we have

$$
\frac{f_{i}(z)}{z}=p_{i}(z)
$$

where $p_{i}(z) \in \mathscr{P}$ for all $i=1, \ldots, n$. Differentiating both sides of (27) logarithmically, we obtain

$$
\frac{z f_{i}^{\prime}(z)}{f_{i}(z)}-1=\frac{z p_{i}^{\prime}(z)}{p_{i}(z)}, \quad(i=1, \ldots, n) .
$$

Define the regular function $h(z)$ as in (15). Thus from (28) and (17), we have

$$
\frac{z h^{\prime \prime}(z)}{h^{\prime}(z)}=\sum_{i=1}^{n}\left[\gamma_{i}+\zeta_{i}\right]\left(\frac{z f_{i}^{\prime}(z)}{f_{i}(z)}-1\right), \quad z \in \mathcal{U} .
$$

Form the hypothesis (26) and (29), we immediately have

$$
\left|\frac{z h^{\prime \prime}(z)}{h^{\prime}(z)}\right| \leq \frac{(2 a+1)^{(2 a+1) / 2 a}}{2}
$$

for all $z \in \mathscr{U}$. Applying Lemma 6, we obtain

$$
\left|\frac{z h^{\prime \prime}(z)}{h^{\prime}(z)}\right| \leq \frac{(2 a+1)^{(2 a+1) / 2 a}}{2}|z|, \quad z \in \mathcal{U} \text {. }
$$

Thus from (29) and (31) we have,

$$
\frac{1-|z|^{2 a}}{a}\left|\frac{z h^{\prime \prime}(z)}{h^{\prime}(z)}\right| \leq \frac{|z|\left(1-|z|^{2 a}\right)}{a} \cdot \frac{(2 a+1)^{(2 a+1) / 2 a}}{2},
$$

for all $z \in \mathcal{U}$. Let us denote $|z|=x, x \in[0,1], \operatorname{Re}(\alpha)=a>0$, and $\Psi(x)=x\left(1-x^{2 a}\right)$. It is easy to prove that the maximum is attained at the point $x=1 /(2 a+1)^{1 / 2 a}$, and thus we have

$$
\Psi(x) \leq \frac{2 a}{(2 a+1)^{(2 a+1) / 2 a}} .
$$

In view of this inequality and (32), we obtain

$$
\frac{1-|z|^{2 a}}{a}\left|\frac{z h^{\prime \prime}(z)}{h^{\prime}(z)}\right| \leq 1 \quad(z \in \mathcal{U}) .
$$

Applying Lemma 2 for the function $h(z)$, we prove that $B_{\beta}(z) \in \mathcal{S}$.

Letting $n=1, \gamma_{1}=\gamma, \zeta_{1}=\zeta$, and $f_{1}=f$ in Theorem 10, we have the following corollary.

Corollary 11. Let $f(z) \in \mathscr{A}$ satisfies $\operatorname{Re}(f(z)) / z>0$, and

$$
\left|(\gamma+\zeta)\left(\frac{z f^{\prime}(z)}{f(z)}-1\right)\right| \leq \frac{(2 a+1)^{(2 a+1) / 2 a}}{2}
$$

where $\gamma, \zeta, \alpha \in \mathbb{C}$ with $\operatorname{Re}(\alpha)=a>0$, then the integral operator $B_{\beta, \gamma, \zeta}(z)$ defined by (24) is in the class $\mathcal{S}$.

Using Lemma 3, we derive the following theorem.

Theorem 12. Suppose that each of the functions $f_{i}(z) \in \mathscr{A}$ satisfies $\operatorname{Re}\left(\left(f_{i}(z)\right) / z\right)>0$, and

$$
\left|\left(\gamma_{i}+\zeta_{i}\right)\left(\frac{z f_{i}^{\prime}(z)}{f_{i}(z)}-1\right)\right| \leq \frac{|\beta|}{n}(1-|c|),
$$

for all $i=1, \ldots, n$, where $\gamma_{i}, \zeta_{i}, \beta \in \mathbb{C}(\operatorname{Re}(\beta)>0)$ and $c \in$ $\mathbb{C}(|c|<1)$, then the integral operator $B_{\beta}(z)$ defined by (3) is in the class $\mathcal{S}$. 
Proof. From (29), we have

$$
\begin{aligned}
\left.|c| z\right|^{2 \beta} & +\left(1-|z|^{2 \beta}\right) \frac{z h^{\prime \prime}(z)}{\beta h^{\prime}(z)} \mid \\
& =\left.|c| z\right|^{2 \beta}+\frac{\left(1-|z|^{2 \beta}\right)}{\beta} \sum_{i=1}^{n}\left(\gamma_{i}+\zeta_{i}\right)\left(\frac{z f_{i}^{\prime}(z)}{f_{i}(z)}-1\right) \mid \\
& \leq|c|+\frac{1}{|\beta|} \sum_{i=1}^{n}\left|\gamma_{i}+\zeta_{i}\right|\left|\frac{z f_{i}^{\prime}(z)}{f_{i}(z)}-1\right| .
\end{aligned}
$$

Now by using the hypothesis (36), we obtain

$$
\left.|c| z\right|^{2 \beta}+\left(1-|z|^{2 \beta}\right) \frac{z h^{\prime \prime}(z)}{\beta h^{\prime}(z)} \mid \leq 1 .
$$
$\mathcal{S}$.

Finally, by applying Lemma 3, we conclude that $B_{\beta}(z) \epsilon$

Letting $n=1, \gamma_{1}=\gamma, \zeta_{1}=\zeta$, and $f_{1}=f$ in Theorem 12, we have the following corollary.

Corollary 13. Suppose that the functions $f(z) \in \mathscr{A}$ satisfy $\operatorname{Re}(f(z) / z)>0$, and

$$
\left|(\gamma+\zeta)\left(\frac{z f^{\prime}(z)}{f(z)}-1\right)\right| \leq|\beta|(1-|c|),
$$

where $\gamma, \zeta, \beta \in \mathbb{C}(\operatorname{Re}(\beta)>0)$ and $c \in \mathbb{C}(|c|<1)$, then the integral operator $B_{\beta, \gamma, \zeta}(z)$ defined by $(24)$ is in the class $\mathcal{S}$.

\section{Acknowledgment}

The author would like to thank the referee for his helpful comments and suggestions.

\section{References}

[1] B. A. Frasin and M. Darus, "On certain analytic univalent functions," International Journal of Mathematics and Mathematical Sciences, vol. 25, no. 5, pp. 305-310, 2001.

[2] B. A. Frasin, "A note on certain analytic and univalent functions," Southeast Asian Bulletin of Mathematics, vol. 28, no. 5, pp. 829-836, 2004.

[3] B. A. Frasin and Á. Baricz, "Univalence of integral operators involving Bessel functions," Applied Mathematics Letters, vol. 23, no. 4, pp. 371-376, 2010.

[4] D. Blezu and R. N. Pascu, "Univalence criteria for integral operators," Glasnik Matematički. Serija III, vol. 36, no. 56, pp. 241-245, 2001.

[5] D. Breaz and N. Breaz, "Univalence conditions for certain integral operators," Studia Universitatis Babeş-Bolyai. Mathematica, vol. 47, no. 2, pp. 9-15, 2002.

[6] D. Breaz and V. Pescar, "Univalence conditions for some general integral operators," Banach Journal of Mathematical Analysis, vol. 2, no. 1, pp. 53-58, 2008.
[7] S. Bulut, "Univalence preserving integral operators defined by generalized Al-Oboudi differential operators," Analele stiintifice ale Universitatii Ovidius Constanta, vol. 17, no. 1, pp. 37-50, 2009.

[8] B. A. Frasin, "Univalence of two general integral operator," Filomat, vol. 23, no. 3, pp. 223-229, 2009.

[9] B. A. Frasin, "Order of convexity and univalency of general integral operator," Journal of the Franklin Institute, vol. 348, no. 6, pp. 1013-1019, 2011.

[10] B. A. Frasin, "New general integral operator," Computers \& Mathematics with Applications, vol. 62, no. 11, pp. 4272-4276, 2011.

[11] B. A. Frasin, "New criteria for univalence of certain integral operators," Acta Mathematica. Academiae Paedagogicae Nyíregyháziensis, vol. 27, no. 1, pp. 31-39, 2011.

[12] G. I. Oros, G. Oros, and D. Breaz, "Suffcient conditions for univalence of an integral operator," Journal of Inequalities and Applications, vol. 2008, Article ID 127645, 7 pages, 2008.

[13] V. Pescar, "Univalence of certain integral operators," Acta Universitatis Apulensis, no. 12, pp. 43-48, 2006.

[14] V. Pescar, "On some integral classes of integral operators," General Mathematics, vol. 16, no. 1, pp. 11-17, 2008.

[15] V. Pescar, "Univalence conditions for certain integral operators," Journal of Inequalities in Pure and Applied Mathematics, vol. 7, no. 4, article 147, 6 pages, 2006.

[16] D. Breaz and N. Breaz, "Two integral operators," Studia Universitatis Babes-Bolyai, Mathematica, Cluj-Napoca, vol. 47, no. 3, pp. 13-21, 2002.

[17] B. A. Frasin, "Integral operator of analytic functions with positive real part," Kyungpook Mathematical Journal, vol. 51, no. 1, pp. 77-85, 2011.

[18] N. N. Pascu, "An improvement of Becker's univalence criterion," in Proceedings of the Commemorative Session: Simion Stoilow, pp. 43-48, University of Brasov, Braşov, Romania, 1987.

[19] T. H. MacGregor, "The radius of univalence of certain analytic functions," Proceedings of the American Mathematical Society, vol. 14, pp. 514-520, 1963.

[20] E. Deniz and H. Orhan, "An extension of the univalence criterion for a family of integral operators," Annales Universitatis Mariae Curie-Skłodowska. Sectio A, vol. 64, no. 2, pp. 29-35, 2010.

[21] Z. Nehari, Conformal Mapping, Dover, New York, NY, USA, 1975. 


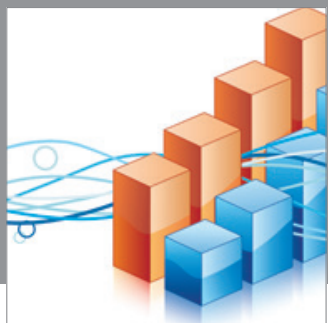

Advances in

Operations Research

mansans

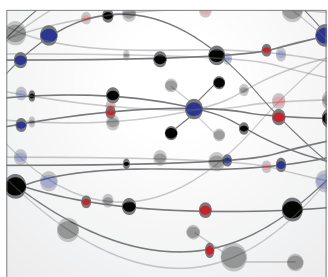

The Scientific World Journal
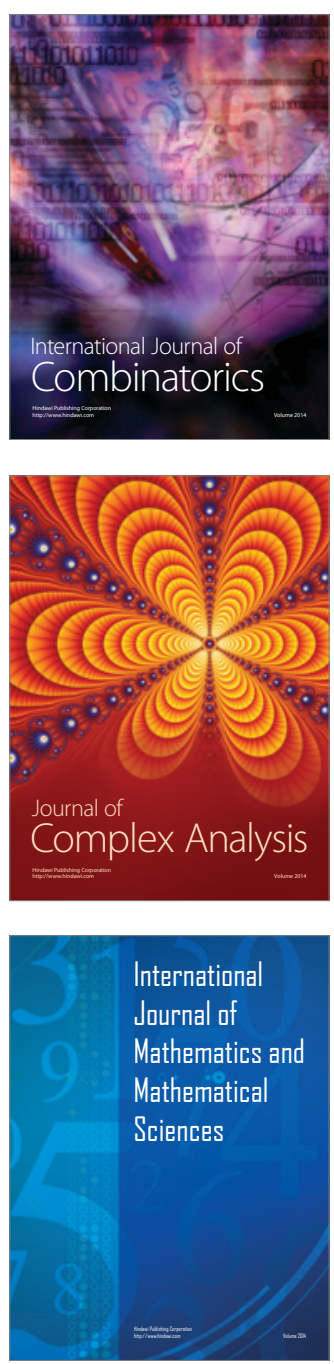
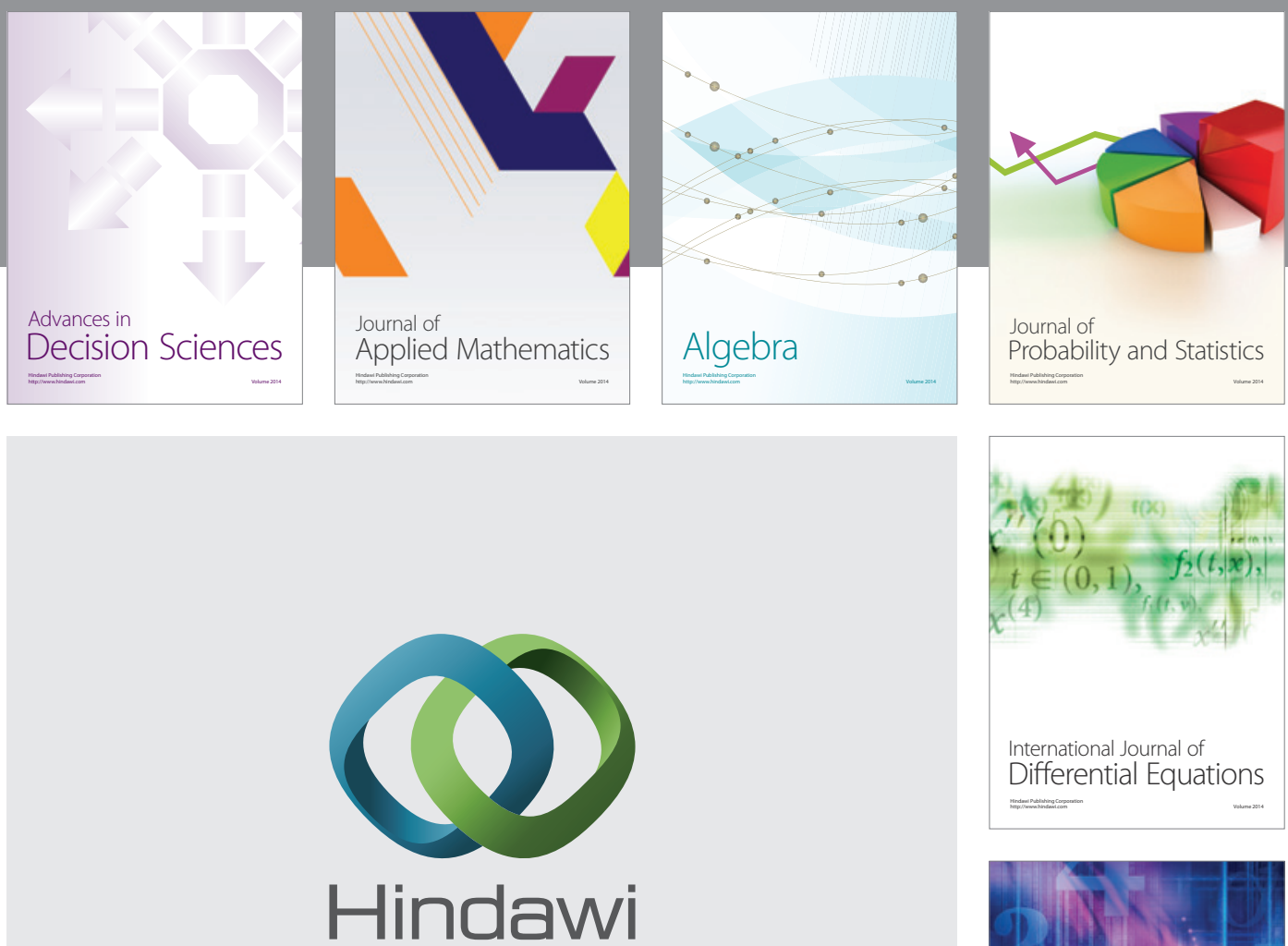

Submit your manuscripts at http://www.hindawi.com
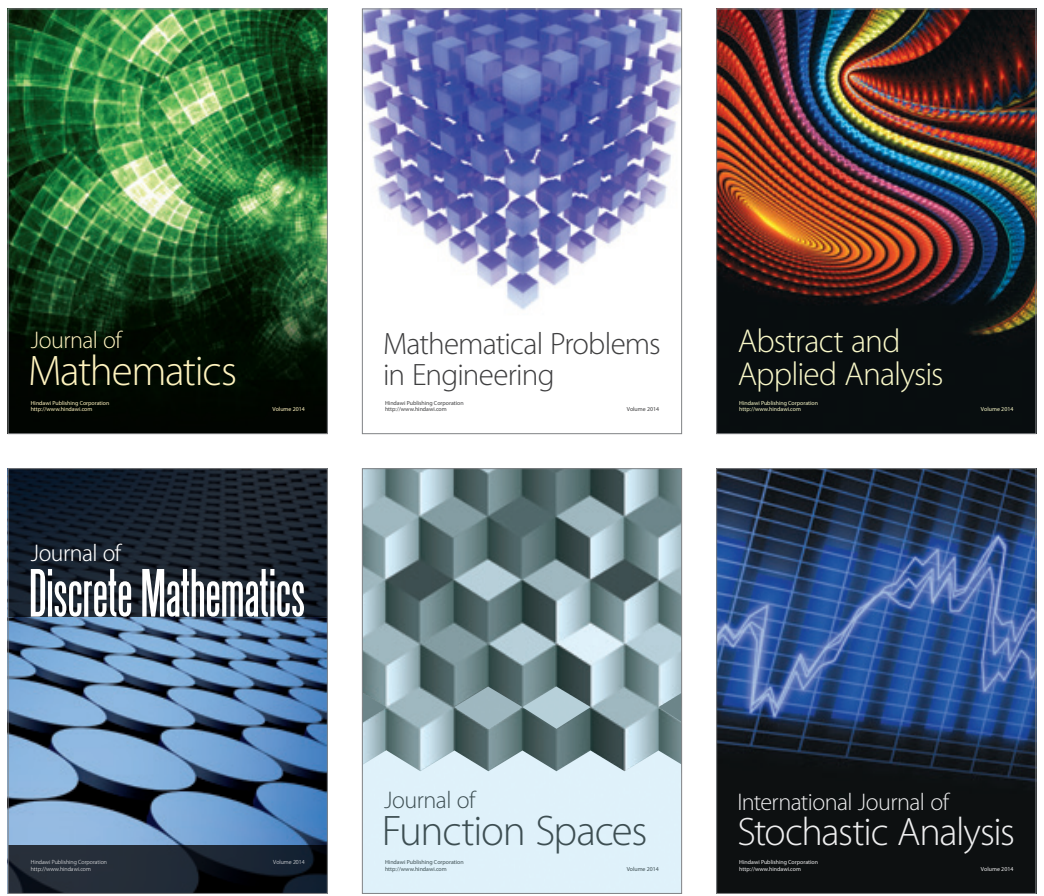

Journal of

Function Spaces

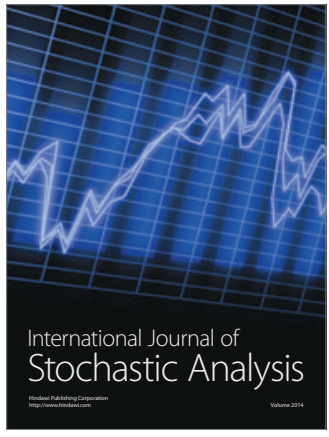

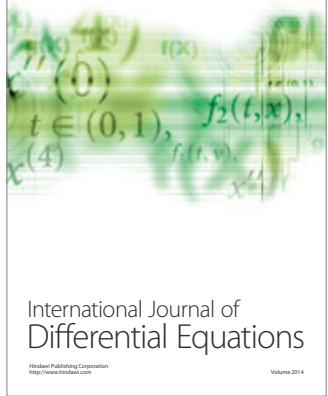
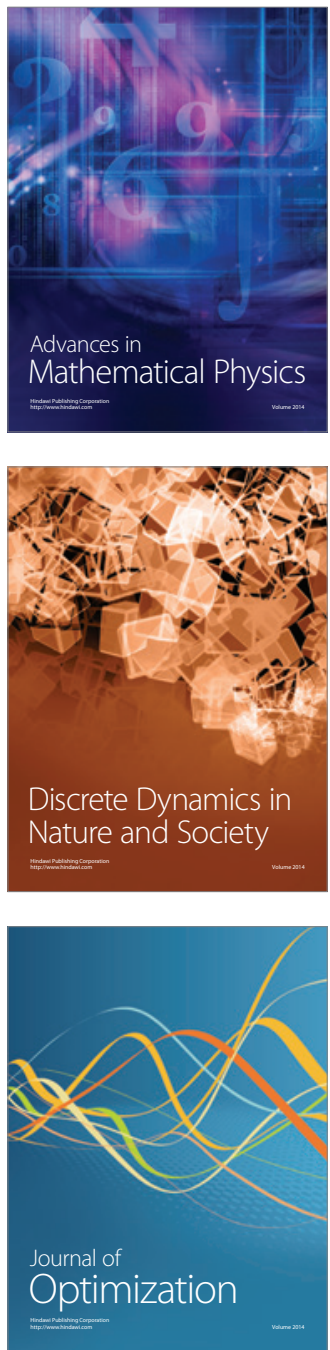